\title{
Cloning, molecular characterization, and expression analysis of a nucleoporin gene (rgNUP98-96) from Rehmannia glutinosa
}

\author{
Y.H. Yang' and M.J. $\mathbf{L i}^{2}$ \\ ${ }^{1}$ College of Bioengineering, Henan University of Technology, Zhengzhou, China \\ ${ }^{2}$ College of Crop Sciences, Fujian Agriculture and Forestry University, Fuzhou, China \\ Corresponding author: Y.H. Yang \\ E-mail: yyhui2004@126.com \\ Genet. Mol. Res. 14 (4): 13022-13032 (2015) \\ Received April 30, 2015 \\ Accepted August 19, 2015 \\ Published October 21, 2015 \\ DOI http://dx.doi.org/10.4238/2015.October.21.23
}

ABSTRACT. Nucleoporin 98 (NUP98) and nucleoporin 96 (NUP96) are essential components of the nuclear pore complex (NPC) in eukaryote cells. However, there is a lack of available information about complete Rehmannia glutinosa NUP98-96 (rgNUP98-96) sequences. Here, the full-length cDNA sequence of rgNUP96-98 was isolated from $R$. glutinosa using rapid amplification of CDNA ends (RACE) technology, based on a cloned cDNA sequence (GenBank accession No. JZ483329). The identified rgNUP98-96 was $3476 \mathrm{bp}$, and it encoded a 1041-amino acid peptide. The BLAST search analysis of rgNUP98-96 showed an intermediate degree of similarity $(60-79 \%)$ to the NUP98-96 protein sequences of 34 other plants, including the dicotyledons Erythranthe guttata, Genlisea aurea, Coffea canephora, Nicotiana benthamiana, Solanum lycopersicum, and Solanum tuberosum. The phylogenetic analysis of NUP96-98 sequences indicated that $R$. glutinosa and $E$. guttata sequences shared the closest homology. The calculated molecular mass and predicted isolectric point of the complex protein were $117.6 \mathrm{kDa}$ and 4.99 , respectively. The secondary and threedimensional structure studies illustrated that the rgNUP96-98 protein folded into a channel motif comprised of 34 alpha-helices, nine beta-strands, and 
several long loops. Using quantitative real-time PCR, the spatio-temporal expression patterns of rgNUP98-96 were analyzed in R. glutinosa, and the results indicated that $r g N U P 98-96$ was highly expressed at the early stage of $R$. glutinosa tuberous root expansion, which is associated with a higher expression pattern in roots. The study provides a valuable foundation for further investigation of rgNUP96-98 molecular functions in R. glutinosa.

Key words: NUP98-96; R. glutinosa; RACE technology; qRT-PCR; Sequence analysis

\section{INTRODUCTION}

The nuclear pore complex (NPC) is the sole gateway between the nucleoplasm and the cytoplasm, reconciling RNA and protein traffic in eukaryotic cells. It is a crucial process for various cellular activities that regulate gene expression, facilitate cell nucleocytoplasmic transport, and influence development (Fiserova et al., 2009; Hetzer and Wente, 2009; Bonnet et al., 2015). Plant NPCs are comprised of multiple copies of approximately 30 different proteins called nucleoporins (Parry, 2013; 2014). Nucleoporin 98 (NUP98; a gatekeeper) and nucleoporin 96 (NUP96; a scaffold), are essential components of the NPC, and are located at both the cytoplasmic and the nuclear sides of the NPC central channel (Terry and Wente 2009; Parrott et al., 2011; Stuwe et al., 2012; Franks and Hetzer, 2013; Byrne et al., 2014; Morchoisne-Bolhy et al., 2015). In many cells, the conservedNUP9896genes generate a long mRNA with an open reading frame (ORF) for a polyprotein. This polyprotein is processed by autocatalytic cleavage that subsequently separates the two functional units (NUP98 and NUP96), which control the transcription of specific target genes that regulate developmental transitions and the cell cycle (Hülsmann et al., 2012; Panda et al., 2015). The composition and function of the NPCs have been well identified and characterized in vertebrate and yeast cells (Tran and Wente, 2006; Aitchison and Rout, 2012), whereas the status of research aimed at deciphering those of the plant NPCs is limited (Tamura et al., 2010; Chang et al., 2013; Tatout et al., 2014). Although NUP98-96 has been identified and annotated by automated computational analysis for some plant species (e.g., Arabidopsis, Solanum lycopersicum, and Glycine max) (Tamura et al., 2010; Potato Genome Sequencing Consortium, 2011; Chang et al., 2013; Park et al., 2014), there is little information about plant NUP98-96 isolation and characterization.

Rehmannia glutinosa (Scrophulariaceae) is a perennial herbaceous plant. It has various medicinal properties that are associated with its astringent, diuretic, anti-inflammatory, anti-fungal, and anti-radiation characteristics. It is also used in treating liver disorders and diabetes. Because of its specific actions, lack of serious side effects, and lower price, $R$. glutinosa is one of the most popular medicines used in traditional Chinese clinical practice. However, there is currently no information regarding specific $R$. glutinosa molecules or nucleoporins with altered nuclear transport during development processes, and the genomic resources available for $R$. glutinosa are limited (Yang et al., 2013). The rapid amplification of cDNA ends (RACE) technique allows the characterization of the full-length sequence of an RNA transcript found in various tissues, especially those of non-model organisms that lack a full genome sequence (Scotto-Lavino et al., 2006a; 2006b). Here, we described the application of RACE technology to obtain a full-length cDNA encoding NUP98-96 from R. glutinosa (rgNUP98-96). We also analyzed the rgNUP98-96 phylogeny and its structural models to present a three-dimensional (3D) structure, which allowed us to recognize its active sites. Finally, we investigated the expression patterns of this gene in various 
tissues during $R$. glutinosa development stages. The purpose was to reveal the possible functions of rgNUP98-96 and their target proteins during the $R$. glutinosa developmental processes.

\section{MATERIAL AND METHODS}

\section{Plant materials}

The R. glutinosa cultivar "Wen 85-5" was cultured from the Wen Agricultural Institute, Jiaozuo City, Henan Province, China. The plants were grown between April 22, 2013 and November 30,2013 . To clone gene sequences, a mixture of five whole plants (including leaves, stems, and roots) was sampled at the early stage of $R$. glutinosa tuberous root expansion (July 22, 2013). The roots, stems, and leaves from five independent plants were collected every month from May 22 to October 22, 2013 to measure gene expression patterns during the following developmental stages: seedlings (May 22), root elongation (June 22), tuberous root expansion (early, July 22; middle, August 22; and late, September 22), and maturity (October 22).

\section{Total RNA extraction, gene cloning, and sequencing}

The TrizOL reagent (TaKaRa Co., Tokyo, Japan) was used to extract total RNA from each sample, following the manufacturer protocol. For 5'-RACE and 3'-RACE, the primers (Table 1) were designed according to a 786 bp fragment of rgNUP98-96 cDNA, which was obtained from R. glutinosa EST sequences in GenBank (accession No. JZ483329) at the National Center for Biotechnology Information (NCBI) (http://www.ncbi.nlm.nih.gov/dbEST/). For 3' and 5'-RACE, total RNA was incubated with SMARTScribeTM Reverse Transcriptase (Clontech Laboratories, Inc., USA ) and $3^{\prime}$ and 5'-RACE CDS Primer A, respectively. Using touchdown PCR, the first CDNA chain was amplified with an rgNUP98-96 gene-specific primer for $5^{\prime}$ and $3^{\prime}$ ends and the Universal Primer A Mix supplied with the kit. Nested PCR was performed with a gene-specific primer for $5^{\prime}$ and $3^{\prime}$ ends and the short Nested Universal Primer A from the kit. The nested PCR products were separated on a $1.2 \%(\mathrm{w} / \mathrm{v})$ agarose gel, and each of the target DNAs $\left(5^{\prime}\right.$ and $3^{\prime}$ ends with the expected size) were then excised from the gel and purified using a MiniBEST Agarose Gel DNA Extraction Kit Ver. 4.0 (TaKaRa Co., Tokyo, Japan). The purified fragments were cloned into the pMD18-T Vector (Takara, Japan) and transformed into E. coli DH5a cells. After screening, the recombinant plasmids were isolated and purified, and the target DNA was Sanger sequenced in both directions. The full-length cDNA of the gene was obtained by assembling the partial cDNA and the $5^{\prime}$ and $3^{\prime}$ ends. Bioinformatic analyses were performed using DNAman software.

Table 1. The primer sequences used in R. glutinosa RACE PCR and qRT-PCR analyses.

\begin{tabular}{|c|c|c|c|c|c|}
\hline PCR Type & & Primer & Sequence (5' to 3 ') & Site (bp) & $\operatorname{Tm}\left({ }^{\circ} \mathrm{C}\right)$ \\
\hline \multirow[t]{4}{*}{ RACE } & Touchdown PCR & 5 ' end & CAAGACACAGCAACTGAGAAACAAATG & 482 & 65.5 \\
\hline & & 3 ' end & TAGGACTGGTAATGTGGTATCAGCTTC & 180 & 67.1 \\
\hline & Nested PCR & 5 ' end & CAAGACACAGCAACTGAGAAACAAATG & 34 & 65.6 \\
\hline & & 3 ' end & ACCTAGCTTATTATCTTATGCTTCTTC & 384 & 62.2 \\
\hline \multirow[t]{4}{*}{ qRT-PCR } & & NUP98-96 & Forward: CATAAGACAAATGCCGCTCTCA & 107 & 60.5 \\
\hline & & & Reverse: ATAAACAATGCCGCGTCAAC & 311 & 58.7 \\
\hline & & $18 \mathrm{~S}$ rRNA* & Forward: TTCTTAGTTGGTGGAGCGATT & 1281 & 58.9 \\
\hline & & & Reverse: CAGACCTGTTATTGCСTCAAAC & 1435 & 59.5 \\
\hline
\end{tabular}

${ }^{*}$ The primers used to amplify the reference gene (18S rRNA) are also listed. 


\section{rgNUP98-96 sequence analysis and prediction}

The obtained nucleotide sequence was translated using Translate Tool (http://www.expasy. ch/tools/translate/), and the properties of the deduced amino acid sequence were estimated using ProtParam (http://web.expasy.org/protparam/). Predictions for isoelectric point (pl) and molecular mass were made using the entire open read frame (ORF) and the pl/MW tool at Expasy (http:// web.expasy.org/compute_pi/). The prediction of protein localization sites in cells was conducted using the PSORT program (http://psort.hgc.jp), and the secondary structure was determined using PSIpred (http://bioinf.cs.ucl.ac.uk/psipred/) (Herbert and Bemstein, 2000). The 3D structure of the rgNUP98-96 protein was predicted using the CPHmodles 3.2 server (http://www.cbs.dtu.dk/services/ CPHmodels/) and the RasMol program (http://en.wikipedia.org/wiki/RasMol/) (Bernstein, 2000). Conserved domains of the deduced protein sequence were obtained by searching for homologous proteins in different databases using the BLASTp-Basic Local Alignment Search tool at NCBI (http://blast.ncbi.nlm.nih.gov/Blast/). Sequences with the similarities were separated, and multiple alignments and phylogenetic construction were performed using MEGA 4.0 (Kumar et al., 2008).

\section{Quantitative real-time PCR (qRT-PCR) analysis}

Following incubation with RNase-free DNase I, reverse transcription reactions were performed using $5 \mu \mathrm{g}$ of RNA and M-MLV reverse transcriptase (TaKaRa Co., Tokyo, Japan) in accordance with the manufacturer instructions. For qRT-PCR, the primers were designed based on the rgNUP98-96 ORF sequences. A primer pair for 18S ribosomal RNA (GenBank ID: EU787015.1) was used as the inner control. Primers were designed using Beacon Designer 7.0 software (Premier Biosoft International, Palo Alto, CA, USA), and the PCR primers are shown in Table 1. To detect gene expression abundances, qRT-PCR reactions were performed using a BIO-RAD iQ5 real-time PCR detection system (Bio-Rad Laboratories, Inc., USA) and Maxima ${ }^{\text {TM }}$ SYBR Green qPCR Master Mix (2X) (Fermentas, CanadaCo., Ltd., Canada). The reaction mixture $(25 \mu \mathrm{L})$ contained $0.5 \mu \mathrm{M}$ of each primer and the appropriate amounts of enzymes, cDNA, and fluorescent dyes. All runs used a negative control without adding target cDNA, resulting in no detectable fluorescence signal from the reaction. A range of five total cDNA dilutions was tested under the same conditions. Amplification reactions were initiated with a pre-denaturing step at $95^{\circ} \mathrm{C}$ for $10 \mathrm{~s}$, which was followed by denaturing $\left(95^{\circ} \mathrm{C}\right.$ for $\left.5 \mathrm{~s}\right)$, annealing $\left(59^{\circ} \mathrm{C}\right.$ for $\left.10 \mathrm{~s}\right)$, and extension $\left(72^{\circ} \mathrm{C}\right.$ for $\left.15 \mathrm{~s}\right)$ steps for 40 cycles during the second stage. A final stage of 55 to $95^{\circ} \mathrm{C}$ was used to determine the dissociation curves of the amplified products. All reactions were performed with at least three replicates. The relative expression levels of these genes were calculated using the $2^{-\triangle \Delta C T}$ method, and the data were normalized based on $18 S$ ribosomal RNA CT values (Livak and Schmittgen, 2001).

\section{RESULTS}

\section{Analysis of nucleotides and deduced amino acids}

In order to characterize the full-length rgNUP98-96 cDNA, total RNA was isolated at the early stage of $R$. glutinosa tuberous root expansion. We designed 5 ' and $3^{\prime}$ cDNA touchdown nested PCR primers based on the EST sequence from NCBI Genbank (accession No. JZ483329). Based on the SMART-RACE technology, $2300 \mathrm{bp}$ of $5^{\prime}$ and $1400 \mathrm{bp}$ of $3^{\prime}$ rgNUP98-96 cDNA products were amplified via the touchdown PCR approach (Figure 1a), and approximately 2000 
bp and 1200 bp fragments of rgNUP98-96 5' and 3' cDNA were isolated using the nested PCR method, respectively (Figure 1b). Nested PCR fragments of the expected size were purified, cloned, transformed, and positively screened. Using Sanger sequencing technology, we obtained 1925 bp and 1201 bp of rgNUP98-96 cDNA 5' and 3' ends, respectively. After the sequences were assembled using DNAman, a 3476 bp fragment of the rgNUP98-96 cDNA was generated, containing a 3123 bp coding sequence, which encoded a 1041 amino acid residue protein (Figure 2). Sequence analysis revealed an ATG start codon at a position equivalent to a short (77 bp) 5'-untranslated region (UTR). The framed ATG codon region was also present in the nucleotides at positions $+4(G)$ and $-3(A)$, which was a typical KOAZK (AXXATGG) character sequence from the cap that contained TATA-like,CAAT-like, and GC-Box sequenceelements in the $5^{\prime}$ upstream UTR. In addition, the $3^{\prime}$ downstream UTR existed in an unsteady signal sequence. The results showed that the cDNA was a full-length NUP98-96 sequence that contained a complete ORF.

The calculated molecular mass of the deduced polypeptide was $117.6 \mathrm{kDa}$, and the predicted pl was 4.99 (Table S1). The total number of negatively (Asp + Glu) and positively (Arg + Lys) charged residues were 157 and 102, respectively. Using the PSORT program, the protein subcellular localization prediction of rgNUP98-96 is in the cell nucleus.

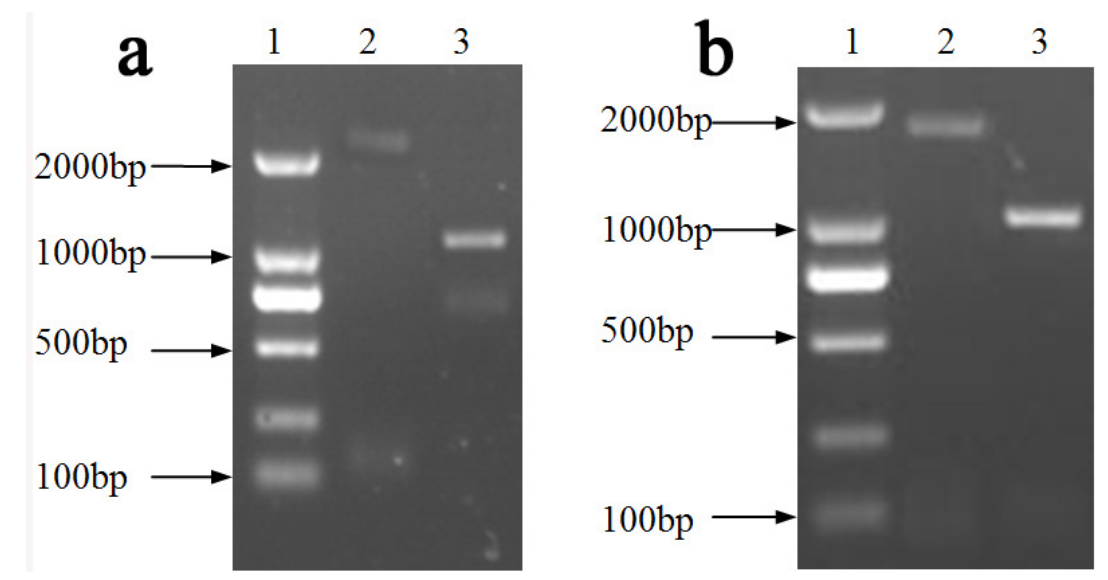

Figure 1. Agarose gel electrophoresis of R. glutinosa NUP98-96 PCR products using SMARTerTM Race technology. (a) Touchdown PCR products. Lane 1: DL2000 size marker; Lane 2: 5' Race PCR products; Lane 3: 3' Race PCR products. (b) Nested PCR products. Lane 1: DL2000 size marker; Lane 2: 5' Race PCR products; Lane 3: 3' Race PCR products.

\section{Analysis of conserved domains and phylogeny}

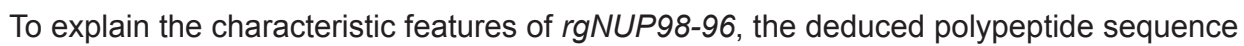
was aligned with the $\mathrm{NCBI}$ non-redundant sequences $(\mathrm{Nr})$ database using BLASTp with default parameters. We detected two putative NUP2 (pfam accession: PF04096) and NUP96 (pfam accession: PF12110) conserved domains (Nup2 and Nup96) in Nup98-96. The rgNUP98-96 polypeptide domain exists in the conserved cleavage site (187) between His-Phe-Ser (HFS) within the NUP2 domain, and it produces two nucleoporins, NUP98 (186 aa) and NUP 96 (855 aa) (Figure 2 and Figure S1).

The deduced rgNUP98-96 amino acid sequence showed an intermediate degree of similarity (60-79\% identity) with the NUP98-96 sequences from 34 other plant species (Table 2). 
To analyze the rgNUP98-96 phylogenetic relationships across plant species, a phylogenetic tree was constructed from an alignment of the rgNUP98-96 deduced amino acid sequence and the sequences from the 34 plant species (Figure 3). As expected, the NUP98-96 sequences of $R$. glutinosa and six other dicotyledons (Erythranthe guttata, Genlisea aurea, Coffea canephora, Nicotiana benthamiana, Solanum lycopersicum, and Solanum tuberosum), indicated close homology, and the sequence relationships were especially close between $R$. glutinosa and Erythranthe guttata.

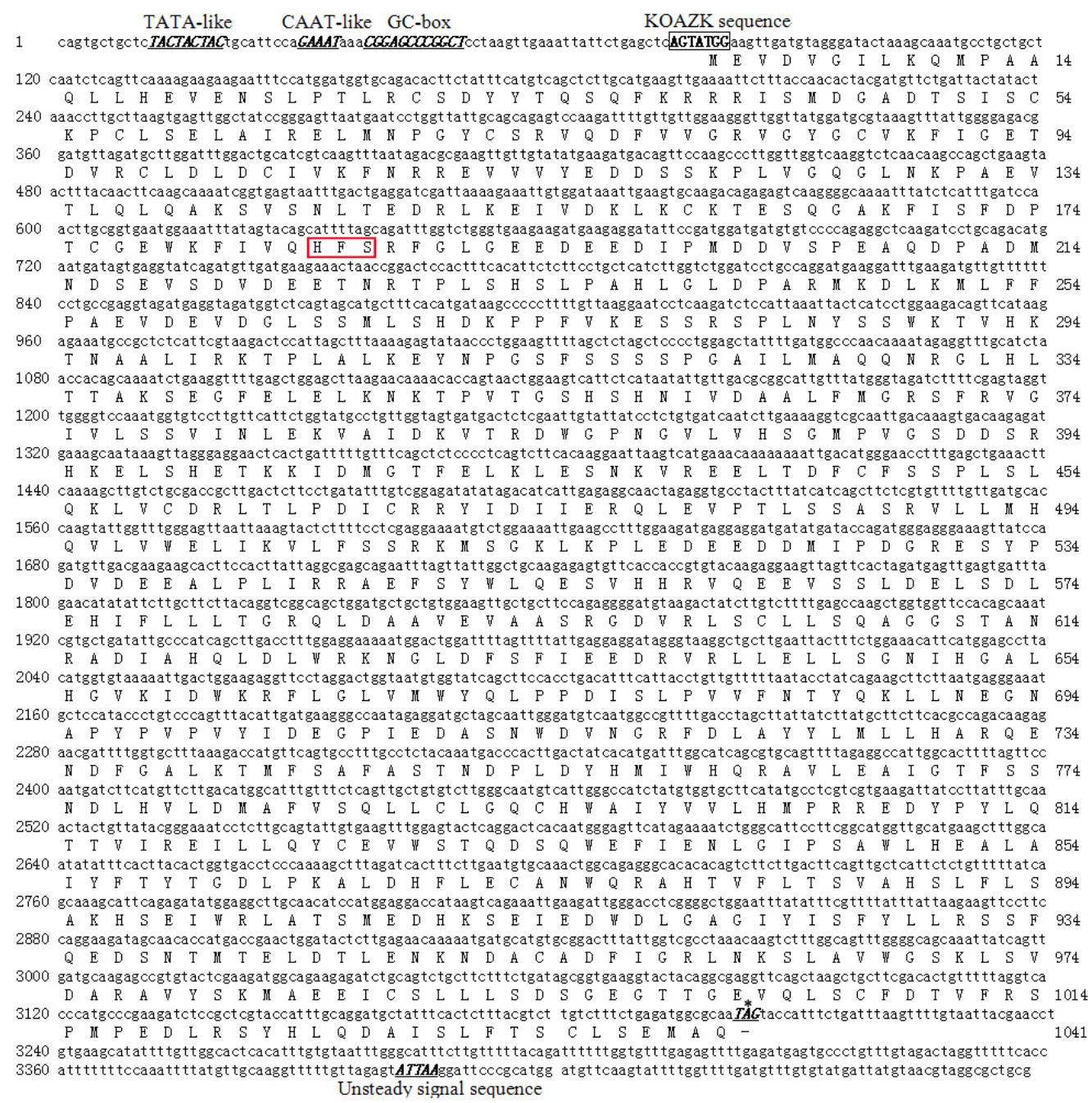

Figure 2. Full-length $R$. glutinosa rgNUP98-96 cDNA sequences and deduced amino acids. Note: The cDNA letters and numbersare in black font and those for proteins are in blue font. rgNUP98-96 has three 5'-UTR characters (including TATA-like, CAAT-like, and GC-box sequence fragments) at the beginning of the 5 ' end cDNA, which are shown in boldface and underlined. The KOAZK sequence around the initiation codon is marked in boldface and is highlighted with a black box. The terminator codon is marked with "*", is shown in boldface and underlined. An unsteady signal sequence (ATTAA) from the 3'-UTR is shown in boldface and underlined. A cleavage motif from the deduced amino acid is highlighted with a red box. 
Table 2. The rgNUP98-96 gene identity analysis between $R$. glutinosa and other plants.

\begin{tabular}{|c|c|c|c|c|}
\hline Plant species & Total score & Query cover (\%) & Identity (\%) & Accession \\
\hline Arabidopsis lyrata subsp. lyrata & 1256 & 98 & 61 & XP_002889279.1 \\
\hline Arabidopsis thaliana & 1263 & 98 & 61 & NP_178183.2 \\
\hline Capsella rubella & 1247 & 98 & 61 & XP_006300673.1 \\
\hline Cicer arietinum & 1265 & 99 & 61 & XP_004491602.1 \\
\hline Citrus clementina & 1369 & 98 & 64 & XP_006427580.1 \\
\hline Citrus sinensis & 1354 & 98 & 64 & XP_006465209.1 \\
\hline Coffea canephora & 1456 & 94 & 72 & CDO98208.1 \\
\hline Cucumis melo & 1285 & 98 & 61 & XP_008449614.1 \\
\hline Cucumis sativus & 1281 & 99 & 61 & XP_004140177.1 \\
\hline Eucalyptus grandis & 1303 & 98 & 63 & KCW70788.1 \\
\hline Eutrema halophilum & 1257 & 98 & 61 & BAJ34529.1 \\
\hline Eutrema salsugineum & 1259 & 98 & 61 & XP_006389822.1 \\
\hline Fragaria vesca subsp. vesca & 1274 & 95 & 62 & XP_004301585.1 \\
\hline Genlisea aurea & 1317 & 95 & 66 & EPS71530.1 \\
\hline Glycine max & 1286 & 99 & 60 & XP_003544079.1 \\
\hline Jatropha curcas & 1397 & 98 & 66 & KDP41997.1 \\
\hline Malus domestica & 1014 & 65 & 70 & XP_008357609.1 \\
\hline Medicago truncatula & 1231 & 99 & 60 & XP_003618002.1 \\
\hline Erythranthe guttatus & 1670 & 100 & 79 & EYU21718.1 \\
\hline Morus notabilis & 1150 & 85 & 62 & EXB67202.1 \\
\hline Nicotiana benthamiana & 1484 & 99 & 69 & BAO49746.1 \\
\hline Oryza stativa Indica group & 1113 & 95 & 60 & EAY88730.1 \\
\hline Oryza stativa Japonica group & 1108 & 95 & 60 & NP_001049116.1 \\
\hline Phaseolus vulgaris & 1276 & 99 & 61 & XP_007142513.1 \\
\hline Phoenix dactylifera & 1239 & 98 & 61 & XP_008801016.1 \\
\hline Prunus mume & 1365 & 99 & 64 & XP_008232110.1 \\
\hline Prunus persica & 1371 & 99 & 64 & XP_007220283.1 \\
\hline Prunus trichocarpa & 1365 & 98 & 65 & XP_002303836.1 \\
\hline Ricinus communis & 1345 & 99 & 63 & XP_002527319.1 \\
\hline Solanum lycopersicum & 1471 & 99 & 69 & XP_006341370.1 \\
\hline Solanum tuberosum & 1429 & 99 & 67 & XP_004235924.1 \\
\hline Theobroma cacao & 1408 & 98 & 65 & XP_007023385.1 \\
\hline Vitis vinifera & 1471 & 98 & 69 & XP_002271967.1 \\
\hline Zea mays & 619 & 57 & 60 & XP_008676303.1 \\
\hline
\end{tabular}

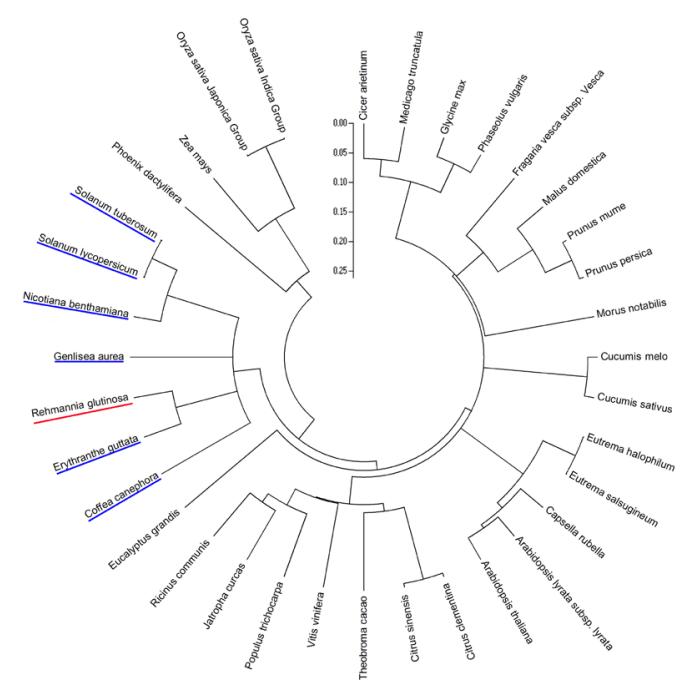

Figure 3. Phylogenetic analysis of the rgNUP98-96 deduced amino acid sequence with the sequences of other plants. The scale over the tree refers to the divergence percentage. 


\section{rgNUP98-96 secondary structure prediction}

Secondary structure analysis of the rgNUP98-96 protein by SOMPA and PSIpred programs revealed that it consisted of 34 alpha-helices and nine beta-strands (Figure 4). Using the CPHmodels 3.2 server and the RasMol program, the 3D structure of rgNUP98-96 (Figure 5) was determined using the crystal structure of the $\mathrm{C}$-terminal autoproteolytic domain of the human nucleoporin NUP98 (PDB ID code 1KO6.A) as a template for comparative modeling. An estimated $42.3 \%$ identity was calculated between human and R. glutinosa NUP98-96 aligned regions (E value $=1 \mathrm{e}-19)$. The $3 \mathrm{D}$ structure of the rgNUP98-96 protein from front or back views indicated that it roughly folded into similar channel-pore motifs with closely surrounding helices. The 3D structure from left and right views is somewhat similar to a half-open sandwich with some helices.

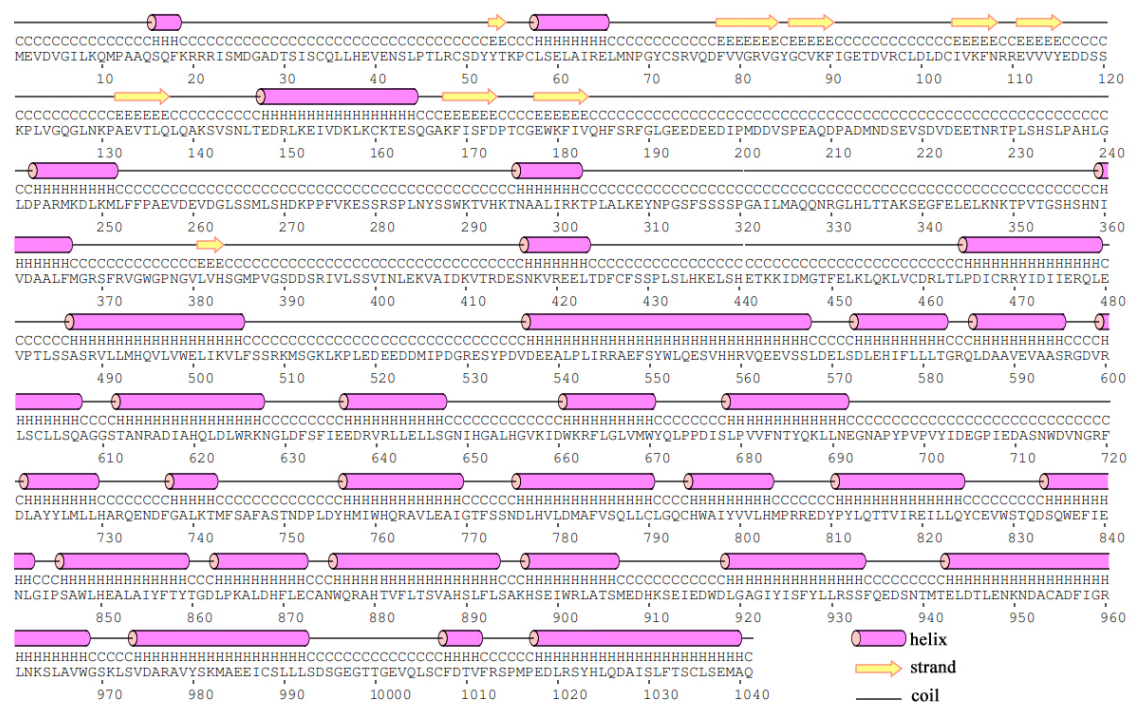

Figure 4. Predicted secondary structure of rgNUP98-96 using the PSIpred program.
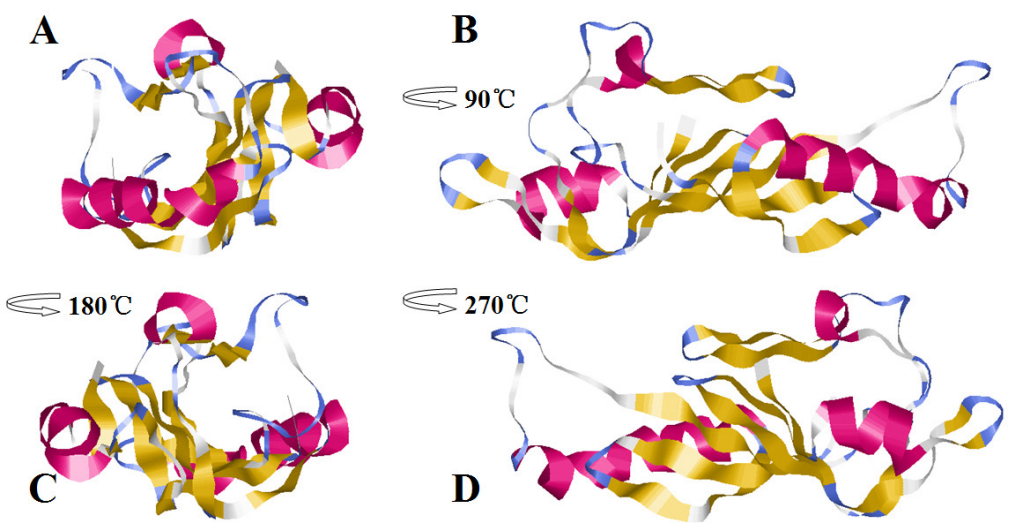

Figure 5. Cartoon display of the 3D structure of rgNUP98-96. The front, left, back, and right views are represented as $A, B, C$, and $D$, respectively. 


\section{Expression patterns of rgNUP98-96}

Using qRT-PCR analyses, the expression levels of rgNUP98-96 were compared between the roots, stems, and leaves of $R$. glutinosa at six different development stages (Figure 6). The results indicated that rgNUP98-96 displayed some differential expression in the roots, stems, and leaves at the six stages. The accumulation levels of the rgNup98-96 transcripts in roots were higher than in stems and leaves for three stages of $R$. glutinosa development, and it was strongly transcribed at the early stage of tuberous root expansion. In contrast, there was almost no tissue-specific expression at the seedling, late tuberous root expansion, and maturity stages. Moreover, rgNUP98-96 is essentially not expressed in tissues during the maturity stage of $R$. glutinosa development.

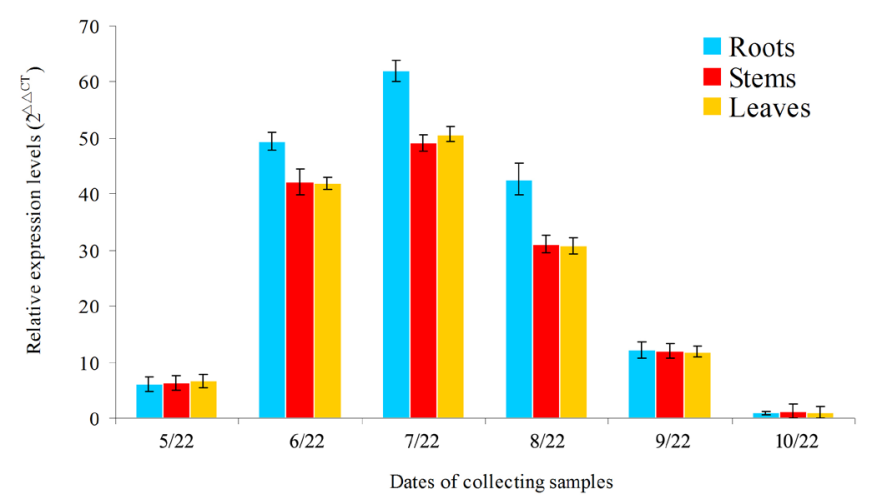

Figure 6. Expression patterns of rgNUP98-96 in R. glutinosa roots, stems, and leaves at six developmental stages.

\section{DISCUSSSION}

While most plant NUP cDNA sequences were obtained through searching available genomes (Parry, 2013; 2014), few NUP genes from plants without genomes have been characterized by the methods used in this study. Here, due to the lack of an R. glutinosa genome (Yang et al., 2013), it was necessary to successfully isolate and characterize rgNUP98-96 using RACE and bioinformatics technologies.

Previous reports indicated that the aminoacid length of NUP98-96 ranged from approximately 950-1100 aa in other most plant species (Alverson et al., 2011; Motamayor et al., 2013; Mybury et al., 2014). In our study, a 3476 bp cDNA fragment with a 3123 bp ORF was isolated from $R$. glutinosa, which was translated into a 1041 aa with a calculated molecular mass of 117.6 kDa. In many eukaryotic cells, NUP98-96 is a conservedand long mRNA, and its ORF encodes a polyprotein that is auto-catalytically cleaved at a conserved site that subsequently separates the NUP98 and NUP96 proteins (Iwamoto et al., 2010). In R. glutinosa, rgNUP98-96 was also a single polypeptide gene, which encoded both NUP98 and NUP96 proteins. Another characteristic feature of the rgNUP98-96 protein were conserved auto-cleaved amino acid residues (HFS) in the C-terminal region (187-189 aa) as previously described in most organisms (Mans et al., 2004; Parrott et al., 2011). The secondary structure analysis of the rgNUP98-96 protein indicated high identity with that of the S. tuberosum NUP98-96 protein, and the rgNUP98-96 3D structure was highly similar to the human NUP98-96, suggesting that the rgNUP98-96 molecular structure and function might be essential characters of most eukaryotic cells. 
Since NUP98 and NUP96 are expressed as a single polypeptide, the amounts of the two nucleoporins in the cell are nearly equivalent. Therefore, we measured the rgNUP98-96 expression patterns in order to examine its regulation in various tissues and at different development stages of $R$. glutinosa. The results indicated that rgNUP98-96 expression levels were highest at the early stage of $R$. glutinosa tuberous root expansion. Therefore, the early stage of tuberous root expansion is a key phase, and the gene expression results are in agreement with previous reports (Yang et al., 2013; 2014). Moreover, we found that rgNUP98-96 expression levels in R. glutinosa roots were higher than that in its stems and leaves at three development stages. It has been reported in several plant species thatNUP98-96 products (NUP98 and NUP96) have a variety of specific functions that regulate cell division and differentiation programs (Parrott et al., 2011; Parry, 2014). We hypothesized that higher rgNUP98-96 expression in $R$. glutinosa roots could be involved in cellular differentiation and division processes, thus promoting tuberous root expansion.

Although the details of the mechanisms underlying these processes require considerable experimental validation, this study could shed light to clarify the molecular basis of rgNUP98-96 functions. In conclusion, the rgNUP98-96 cDNA characterization succeeded in providing a number of valuable clues to aid in understanding the molecular mechanisms of $R$. glutinosa developmental processes. Furthermore, the findings contribute to the broader understanding of basic NPC molecular structure and function in plants.

\section{Conflicts of interest}

The authors declare no conflict of interest.

\section{ACKNOWLEDGMENTS}

Research supported by grants from the National Natural Science Foundation of China (\# 81403037), the Science and Technology Research Key Project of Henan Educational Committee (\#13A180160), and the High-level Personnel Scientific Research Start-up Foundation of Henan University of Technology (\#2013BS035).

\section{Supplementary material}

\section{REFERENCES}

Aitchison JD and Rout MP (2012). The yeast nuclear pore complex and transport through it. Genetics 190: 855-883. Alverson AJ, Rice DW, Dickinson S, Barry K, et al. (2011). Origins and recombination of the bacterial-sized multichromosomal mitochondrial genome of cucumber. Plant Cell 23: 2499-2513.

Bernstein HJ (2000). Recent changes to RasMol, recombining the variants. Trends Biochem. Sci. 25: 453-455.

Bonnet A, Bretes H and Palancade B (2015). Nuclear pore components affect distinct stages of intron-containing gene expression. Nucleic Acids Res. 43: 4249-4261.

Byrne M, Bennett RL, Cheng X and May WS (2014). Progressive genomic instability in the Nup98-HoxD13 model of MDS correlates with loss of the PIG-A gene product. Neoplasia 16: 627-633.

Chang S, Wang Y, Lu J, Gai J, et al. (2013). The mitochondrial genome of soybean reveals complex genome structures and gene evolution at intercellular and phylogenetic levels. PLoS One8: e56502.

Fiserova J, Kiseleva E and Goldberg MW (2009). Nuclear envelope and nuclear pore complex structure and organization in tobacco BY-2 cells. Plant J. 59: 243-255.

Franks TM and Hetzer MW (2013). The role of Nup98 in transcription regulation in healthy and diseased cells. Trends Cell Biol. 23: 112-117. 
Hetzer MW and Wente SR (2009). Border control at the nucleus: biogenesis and organization of the nuclear membrane and pore complexes. Dev. Cell. 17: 606-616.

Hülsmann BB, Labokha AA and Görlich D (2012). The permeability of reconstituted nuclear pores provides direct evidence for the selective phase model. Cell 150: 738-751.

Iwamoto M, Asakawa H, Hiraoka Y and Haraguchi T (2010). Nucleoporin Nup98: a gatekeeper in the eukaryotic kingdoms. Genes Cells 15: 661-669.

Kumar S, Dudley J, Nei M and Tamura K (2008). MEGA: A biologist-centric software for evolutionary analysis of DNA and protein sequences. Brief. Bioinform. 9: 299-306.

LivakKJ and SchmittgenTD (2001). Analysis ofrelati vegene expression data using real-time quantitative PCRandthe2 (-DeltaDeltaC(T)). Method 25: 402-408.

Mans BJ, Anantharaman V, Aravind L and Koonin EV (2004). Comparative genomics, evolution and origins of the nuclear envelope and nuclear pore complex. Cell Cycle 3: 1612-1637.

Morchoisne-Bolhy S, Geoffroy MC, Bouhlel IB, Alves A, et al. (2015). Intranuclear dynamics of the Nup107-160 complex. Mol. Biol. Cell 26: 2343-2356.

Motamayor JC, Mockaitis K, Schmutz J, Haiminen N, et al. (2013). The genome sequence of the most widely cultivated cacao type and its use to identify candidate genes regulating pod color. Genome Biol. 14: r53.

Panda D, Gold B, Tartell MA, Rausch K, et al. (2015). The transcription factor FoxK participates with Nup98 to regulate antiviral gene expression. mBio 6: e02509-14.

Park GT, Frost JM, Park JS, Kim TH, et al. (2014). Nucleoporin MOS7/Nup88 is required for mitosis in gametogenesis and seed development in Arabidopsis. Proc. Natl. Acad. Sci. USA 111: 18393-18398.

Parrott BB, Chiang Y, Hudson A, Sarkar A, et al. (2011). Nucleoporin98-96 function is required for transit amplification divisions in the germ line of Drosophila melanogaster. PLoS One 6: e25087.

Parry G (2013). Assessing the function of theplantnuclear pore complexand the search for specificity. J. Exp. Bot. 64: 833-845.

Parry G (2014). Components of the Arabidopsis nuclear pore complex play multiple diverse roles in control of plant growth. $J$. Exp. Bot. 65: 6057-6067.

Potato Genome Sequencing Consortium (2011). Genome sequence and analysis of the tuber crop potato. Nature 475: 189-195.

Scotto-Lavino E, Du G and Frohman MA (2006a). 5' end cDNA amplification using classic RACE. Nat. Protoc. 1: $2555-2562$.

Scotto-Lavino E, Du G and Frohman MA (2006b). 3' end cDNA amplification using classic RACE. Nat. Protoc. 6: $2742-2745$.

Stuwe T, von Borzyskowski LS, Davenport AM and Hoelz A (2012). Molecular basis for the anchoring of proto-oncoprotein Nup98 to the cytoplasmic face of the nuclear pore complex. J. Mol. Biol. 419: 330-346.

Tamura K, Fukao Y, Iwamoto M, Haraguchi T, et al. (2010). Identification and characterization of nuclear pore complex components in Arabidopsis thaliana. Plant Cell 22: 4084-4097.

Tatout C, Evans DE, Vanrobays E, Probst AV, et al. (2014). The plant LINC complex at the nuclear envelope. Chromosome Res. 22: 241-252.

Terry LJ and Wente SR (2009). Flexible gates: dynamic topologies and functions for FG nucleoporins in nucleocytoplasmic transport. Eukaryotic Cell 8: 1814-1827.

Tran EJ and Wente SR (2006). Dynamic nuclear pore complexes: life on the edge. Cell 125: 1041-1053.

Yang YH, Li MJ, Chen XJ, Wang PF, et al. (2014). De novo characterization of the Rehmannia glutinosa leaf transcriptome and analysis of gene expression associated with replanting disease. Mol. Breeding 34: 905-915.

Yang YH, Zhang ZY, Fan HM, Zhao YD, et al. (2013). Construction and analysis of a different expression cDNA library in Rehmannia glutinosa plants subjected to continuous cropping. Acta Physiol. Plant 35: 645-655. 